\title{
CORRECTIONS
}

\section{Author Correction: Leveraging European infrastructures to access 1 million human genomes by 2022}

Gary Saunders, Michael Baudis, Regina Becker (D), Sergi Beltran, Christophe Béroud, Ewan Birney, Cath Brooksbank, Søren Brunak, Marc Van den Bulcke, Rachel Drysdale, Salvador Capella-Gutierrez (D), Paul Flicek (D), Francesco Florindi, Peter Goodhand, Ivo Gut, Jaap Heringa, Petr Holub (D), Jef Hooyberghs, Nick Juty, Thomas M. Keane, Jan O. Korbel, Ilkka Lappalainen (D), Brane Leskosek, Gert Matthijs, Michaela Th. Mayrhofer (D), Andres Metspalu, Arcadi Navarro, Steven Newhouse, Tommi Nyrönen, Angela Page, Bengt Persson, Aarno Palotie, Helen Parkinson, Jordi Rambla, David Salgado, Erik Steinfelder, Morris A. Swertz, Alfonso Valencia, Susheel Varma $\mathbb{D}$, Niklas Blomberg and Serena Scollen (D)

Nature Reviews Genetics (2019) https://doi.org/10.1038/s41576-019-0156-9 Published online 27 August 2019

The Table 1 (EU declaration signatory and membership status) of the original article published online contained several errors: the IARC was incorrectly listed in the table, which only includes countries, and footnote; Croatia, Lithuania, Poland and Portugal were not listed as full EMBL members; Estonia is now listed as a Prospect Member of EMBL; Hungary, Ireland and Israel were incorrectly listed as BBMRI-ERIC members; Iceland was omitted from the table. Hyphens stand for 'not applicable'; the table and footnote have been corrected accordingly. These errors have now been corrected online and in print.

https://doi.org/10.1038/s41576-019-0178-3 I Published online 13 September 2019 